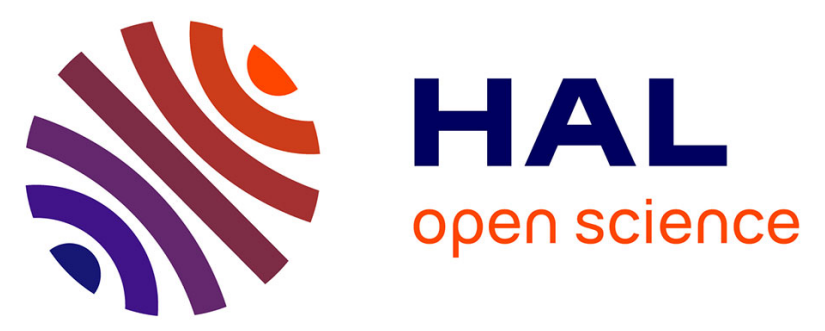

\title{
Near and far-field verification of electro-optic effect enhancement on a tunable lithium niobate photonic crystal.
}

\author{
M.-P. Bernal, M. Roussey, F.I. Baida
}

\section{- To cite this version:}

M.-P. Bernal, M. Roussey, F.I. Baida. Near and far-field verification of electro-optic effect enhancement on a tunable lithium niobate photonic crystal.. Journal of Microscopy, 2008, 229 (2), pp.264-269. 10.1111/j.1365-2818.2008.01897.x . hal-00261575

\section{HAL Id: hal-00261575 \\ https://hal.science/hal-00261575}

Submitted on 30 Apr 2021

HAL is a multi-disciplinary open access archive for the deposit and dissemination of scientific research documents, whether they are published or not. The documents may come from teaching and research institutions in France or abroad, or from public or private research centers.
L'archive ouverte pluridisciplinaire HAL, est destinée au dépôt et à la diffusion de documents scientifiques de niveau recherche, publiés ou non, émanant des établissements d'enseignement et de recherche français ou étrangers, des laboratoires publics ou privés. 


\title{
Near- and far-field verification of electro-optic effect enhancement on a tunable lithium niobate photonic crystal
}

\author{
M.-P. BERNAL, M. ROUSSEY \& F. I. BAIDA \\ Institut FEMTO-ST, Département d'Optique P.M. Duffieux CNRS UMR 6174, Université de Franche \\ Comté 16 Route de Gray, 25030 Besançon Cedex, France
}

Key words. Enhancement of non-linear properties, lithium niobate, photonic crystal, SNOM.

\section{Summary}

In this paper, we present the optical response of a tunable lithium niobate photonic crystal (PC) using the electro-optic effect of the material. The band gap tunability is 300 times higher than what one could expect for a bulk lithium niobate device of the same characteristics. Theoretical calculations based on the finite-difference time domain technique have allowed us to determine the physical origin of this enhanced electro-optic coefficient. Indeed, the effective second-order susceptibility in the LN nanostructure increases, giving rise to an ultra-compact low-voltage photonic crystal modulator when it operates at its band edge. In addition, the theoretical and far-field transmission results are confirmed by nearfield optical microscopy images of the structure at different excitation voltages.

\section{Introduction}

Photonic crystals (PCs), micrometric-sized structures that consist of periodic changes in the dielectric constant, are very attractive optical elements for integrated optics. Apart from the obvious interest that they present owing to their small size, the physical mechanisms that occur inside these nanostructures offer novel optical devices with enhanced properties (Notomi et al., 2001; Schneider \& Watson, 2003; Soljaci \& Joannopoulos, 2004; Vlasov et al., 2005). Indeed, a very interesting feature of PC-based devices on non-linear substrates is the fact that light is strongly localized and, if the operating wavelength is near or at the band edge of the photonic band gap, the effective non-linear susceptibilities of the material can be enhanced because of the giant values of the local field factor inside the photonic structure at those wavelengths. Thus, local field factors of 5 are demonstrated for crossed Kerr effects (Razzari et al., 2005), which have direct

Correspondence to: M.-P. Bernal. Tel: +41 216934 304; fax: +41 216934 666; e-mail: maria-pilar.bernal@univ-fcomte.fr applications in optical signal processing. Soljaci et al. (2002) have also shown that slow group velocities can dramatically increase the induced phase shifts caused by small changes in the index of refraction, making an important step towards large-scale all-optical integration.

In this paper, we use a lithium niobate (LN) PC device (Lacour et al., 2005) to strongly enhance its electro-optic effect. The stop band of the PC moves $200 \mathrm{~nm}$ for an external voltage of $80 \mathrm{~V}$, which means that we report on an electro-optic effect 300 times higher than what it would be measured for the equivalent bulk LN device. The device performance as well as its small active area of only $121 \mu \mathrm{m}^{2}$ makes it 1000 times smaller than state-of-the-art conventional electro-optical devices.

We have chosen LN as the active substrate dielectric material because of its multiple physical properties. It is at the same timenon-linear acousto-and electro-optical, and is widely used already in the fabrication of light modulators in the domain of optical telecommunications.

\section{Description of the structure}

Two-dimensional (2D) finite-difference time domain (FDTD) simulations (Roussey et al., 2005) have been performed in order to find the most suitable structure allowing a tunable PC at the telecommunication wavelengths. Thus, we have chosen a square lattice of holes with a period $a=766 \mathrm{~nm}$ and a radius $r=207 \mathrm{~nm}$, resulting in an aspect ratio $r / a=0.27$. The theoretical transmission band for this particular geometry is shown in Fig. 1a. We can observe a band gap between 1400 and $1550 \mathrm{~nm}$ that is separated into two bands by a thin $(\Delta \lambda=5 \mathrm{~nm})$ transmission band. Simulations, not presented in this paper, show that the width and position of the 'peak' depend on the number of rows and the period of the PC. Light is propagating in the $\Gamma X$ direction and only TE polarization is taken into account owing to technological constraints (Roussey et al., 2005). We would like to point out the fact that the APE waveguides are TE polarized, which eases the light coupling into the 

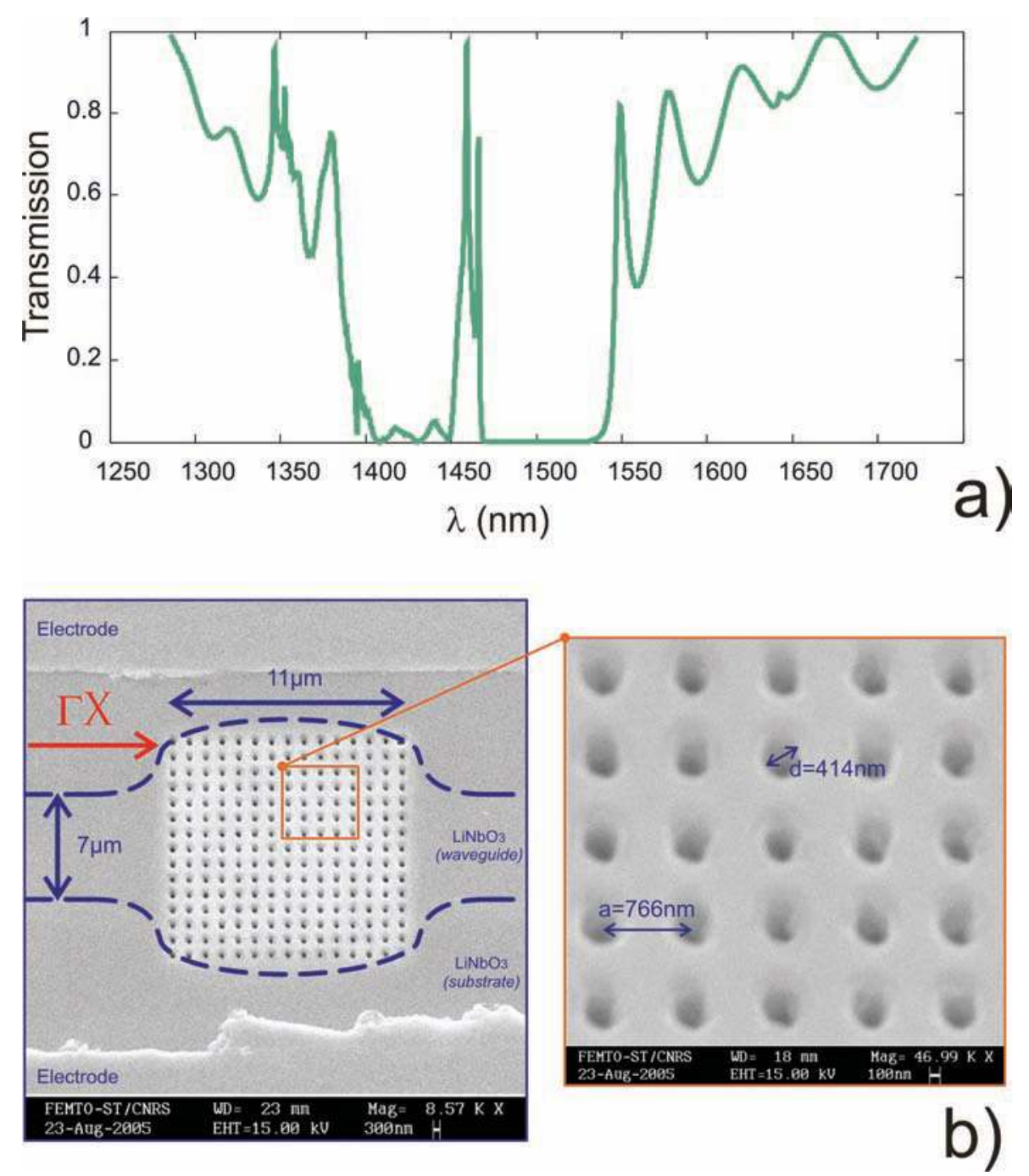

Fig. 1. (a) Simulated transmission spectrum calculated of the square lattice for 15 rows of holes in the propagation direction. (b) SEM images of the photonic crystal. The dashed line, left image, represents the contour of the APE waveguide. The period of the lattice is $a=766 \mathrm{~nm}$ and the radius of the holes is $r=207 \mathrm{~nm}$.

waveguide since there is no need of a polarization control device.

The stop band comprises the wavelengths between 1400 and $1550 \mathrm{~nm}$. After exploring many different PC fabrication techniques that could best apply to $\mathrm{LN}$, and due to its well-known resistance to be etched, focused ion beam (FIB) technique is used to fabricate the PC lattice. A thin layer of chromium is deposited on the substrate in order to avoid charging effects. The PC is then milled using a multi-pass technique to minimize material re-deposition. The hole's depth is around $2 \mu \mathrm{m}$. After the holes' fabrication, the chromium layer is removed with chromium-etch solution. To avoid light leakage into the vertical direction, the PC is fabricated on an LN annealed proton exchange (APE) index gradient waveguide. At a wavelength $\lambda=1.55 \mu \mathrm{m}$, the effective index of refraction of a proton-exchanged LN waveguide is equal to 2.143 , while for the standard material it is 2.138 . The centre of the guided APE mode is located at $1.5 \mu \mathrm{m}$ from the surface and has a depth of $3 \mu \mathrm{m}$. Details of the complete fabrication process can be found in previous works (Lacour et al., 2005; Roussey et al., 2005). The electro-optic effect in the device is achieved by depositing in both sides of the $\mathrm{PC}$, and parallel to the light propagation direction, two titanium electrodes. To use the largest electro-optic coefficient, an X-cut LN wafer is employed.

Figure $1 \mathrm{~b}$ shows SEM images of the fabricated PC device as well as a zoomed SEM image of the PC holes.

\section{Far-field characterization and theoretical explanation}

To observe the entire spectral band range of the PC device and its electro-optic tunability, the device is illuminated with a 


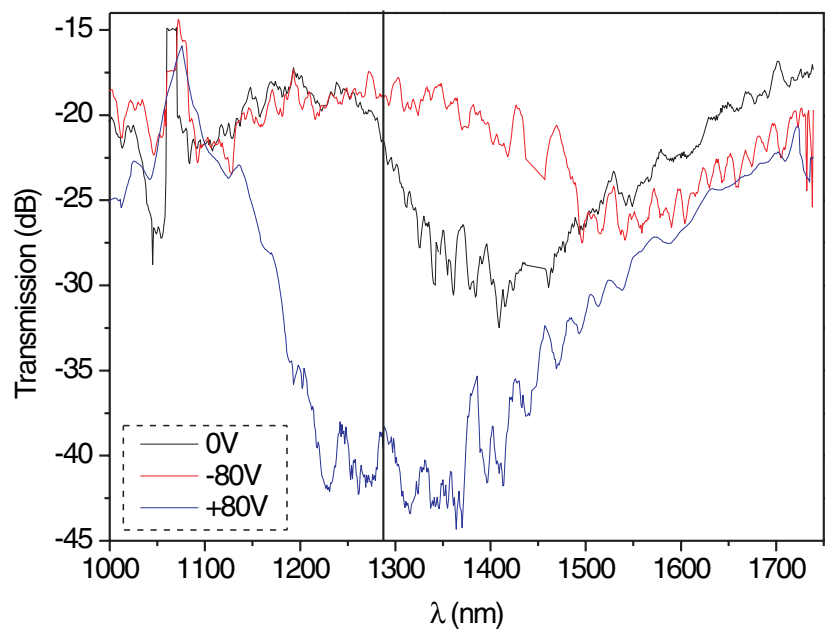

Fig. 2. Experimentally measured transmission spectra at three different voltages ( $0 \mathrm{~V}$ in black, $80 \mathrm{~V}$ in blue and $-80 \mathrm{~V}$ in red).

white light fibre source (Roussey et al., 2005) whose spectral range covers from 600 to $2000 \mathrm{~nm}$. The transmitted light is coupled to a multi-modal optical fibre that is connected to an optical spectrum analyzer.

The measurement of the spectral transmission through the PC has been made at different voltages. Figure 2 presents three spectra corresponding to $-80,0$ and $80 \mathrm{~V}$. The gap in the transmission at $0 \mathrm{~V}$ is larger than the simulated one (Fig. 1a) because of fabrication imperfections coming from the FIB resolution $(50 \mathrm{~nm})$ and from the fact that holes have a conical shape (Bernal et al., 2006). A shift of $200 \mathrm{~nm}$ for an applied voltage of $80 \mathrm{~V}$ is measured at the left band edge of the transmission band, corresponding to a spectrum modulation of $2.5 \mathrm{~nm} \mathrm{~V}^{-1}$. This effect is purely electric since the stopgap translates in the opposite direction as the voltage sign is inverted. To rule out photorefractive effects, the same experiment has been repeated with the sample continuously illuminated during $3 \mathrm{~h}$ by a blue laser beam (output power $=$ $50 \mathrm{~mW}, \lambda=473 \mathrm{~nm}$ ). At this wavelength, photorefractive effect is most significant for LN. However, no additional shift was observed. This value is 312 times bigger than the shift predicted by the Pockels effect on bulk LN of the same size $\left(0.008 \mathrm{~nm} \mathrm{~V}^{-1}\right)$. An estimated increasing of the refractive index of 0.3 has been measured for the wavelength $1286 \mathrm{~nm}$.

To understand these unexpected results, accurate simulations of the light propagation inside the non-linear PC are required. In a bulk material, the well-known classical Pockels equation describing the index of refraction variation with an applied voltage is sufficient. However, these equations are not suitable to describe the influence of a nanostructuration on the non-linear susceptibility of materials like lithium niobate. To consider the nanostructuration, the local field factor $f$ inside the PC must be inserted into the non-linear equations (Dealaye et al., 2005; Razzari et al., 2005).

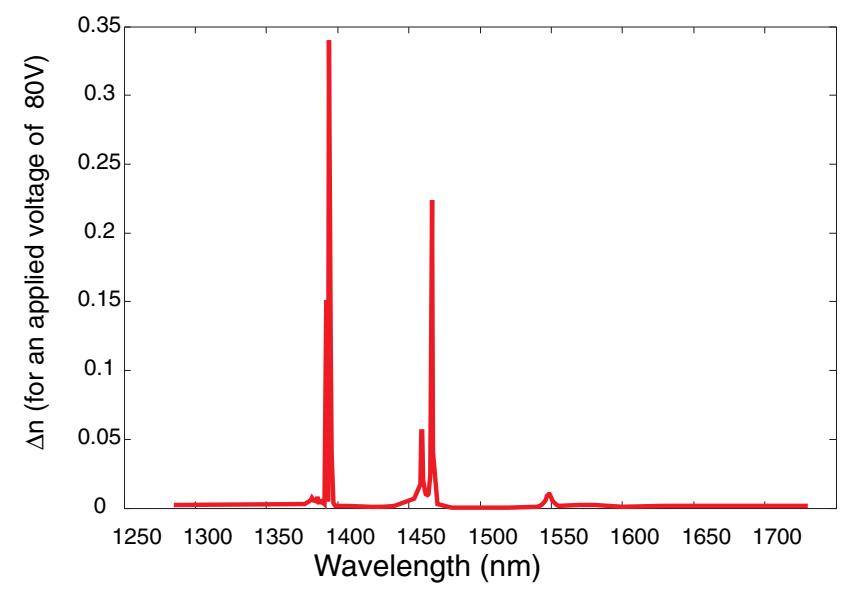

Fig. 3. Calculated value of the index of refraction variation as a function of wavelength.

The effective refractive index difference due to the electrooptic effect can then be described as

$$
\Delta n=-\frac{1}{2} \times n^{3} \times r_{33} \times f^{3} \times E,
$$

where $E$ is the external electric field, $r_{33}$ is the highest electrooptic coefficient and $n$ is the index of refraction. Note that Pockels equation for bulk material is Eq. (1) for $f=1$. The local field factor can be obtained in two different ways (Rigneault et al., 2005).

A first way is by evaluating the group velocities in the bulk material, $v_{\mathrm{g}}^{\mathrm{BULK}}$, and in the PC, $v_{\mathrm{g}}^{\mathrm{PC}}$.

$$
f=\sqrt{\frac{\nu_{\mathrm{g}}^{\mathrm{BULK}}}{v_{\mathrm{g}}^{\mathrm{PC}}} .}
$$

Another way is based on the determination of the field distribution inside the PC, $E_{\mathrm{loc}}^{\mathrm{PC}}$, and to compare it to its value in the bulk substrate, $E_{\mathrm{loc}}^{\mathrm{BUL}}$

$$
f=\frac{1}{\mathrm{NS}} \int_{\mathrm{PC}} \frac{E_{\mathrm{loc}}^{\mathrm{PC}}}{E_{\mathrm{loc}}^{\mathrm{BULK}}} d y d z
$$

where NS is the total PC area.

We can note that when the group velocity in the PC decreases, the local field factor increases and then, according to Eq. (1), the effective refractive index variation increases too. We have calculated the local field factor through Eq. (3) with FDTD simulations. Thus, the electric field in the structure for different wavelengths (in the stop band region) inside the $15 \times$ 15 holes has been calculated. The calculated $\Delta n$ as a function of the wavelength and for an external excitation voltage of $80 \mathrm{~V}$ is shown in Fig. 3, and the calculated optical transmission as a function of the wavelength in Fig. 1a. At the lower band edge of the stop band $(\lambda=1286 \mathrm{~nm})$ and for an external voltage of $80 \mathrm{~V}$, a variation of the index of refraction of 0.33 has been calculated that accurately matches the experimentally measured one $(\Delta n=0.3)$. These results can be interpreted with the fact that 
at the band edges, the group velocity reaches almost a value of 0 , and the $f$ value is increased according to Eq. (2). Light spends more time in the nanostructure that gives an increase in the effective non-linear effect. This slow light effect in PC explains the observed gigantic shift of the gap.

From these results, we can also conclude that this modified Pockels formula is accurate enough to describe the observed phenomenon and that no additional higher-order non-linear terms are needed.

\section{Near-field study}

Scanning near-field optical microscopy (SNOM) is used to support our findings. For this particular case, a near-field characterization of the entire structure at larger scale but keeping the high spatial resolution along the propagation direction is interesting to visualize the optical power distribution and the optical losses and to analyze fabrication problems in the technical process. Although this technique is not systematically studied in PC research, it is of great importance since it allows observing the PC behaviour at scales that are shorter than a wavelength. SNOM technique has already validated slow light phenomena (Gersen et al., 2005) as well as other PC configurations (Gérard et al., 2002; Flück et al., 2003; Koenderik et al., 2005).

Thus, we have utilized a SNOM apparatus to image the light propagation in the lithium niobate tunable PC structure. As in a previous work (Bernal et al., 2006), we have used a commercial scanning near-field optical microscope (NTMDT SMENA) in collection mode. The probe is a dielectricpulled single-mode (at $1550 \mathrm{~nm}$ ) optical fibre. Figure 4 shows the experimental set-up. Laser light at $1286 \mathrm{~nm}$, which corresponds to the left band edge of the stop band at $0 \mathrm{~V}$ is coupled to the optical waveguide. The evanescent field of the PC surface as well as the PC topography are simultaneously recorded.
Figure 5 shows three $15 \times 15-\mu \mathrm{m}$-size optical images of the region where the photonic structure is located. In the three cases, the injected light has the same wavelength, $1286 \mathrm{~nm}$, and the same input power. An intense blotch of light can be observed at the interface between the channel waveguide and the PC. We can attribute this to the impedance mismatch between the waveguide classical mode and the PC operating at the slow light regime. Figure 5 a corresponds to an applied external voltage of $-80 \mathrm{~V}$, Fig. $5 \mathrm{~b}$ to $0 \mathrm{~V}$ and Fig. $5 \mathrm{c}$ to $+80 \mathrm{~V}$. We can observe from the images that the SNOM results agree with the spectral transmission measurements shown in Fig. 2. At $0 \mathrm{~V}, \lambda=1286 \mathrm{~nm}$ falls outside the band gap. At $-80 \mathrm{~V}$, the stop band moves towards the infrared regions and $\lambda=1286 \mathrm{~nm}$ also falls outside the gap. As the voltage increases, the electro-optic effect translates the stop band towards the blue spectra and we can see that $\lambda=1286 \mathrm{~nm}$ falls now inside the stop band.

From the transmission measurements, we should expect to have more transmitted light at $-80 \mathrm{~V}$ than at $0 \mathrm{~V}$. However, the SNOM images show more evanescent light at $-80 \mathrm{~V}$ than $0 \mathrm{~V}$. This is due to the fact that the APE guided mode that propagates through the classical waveguide moves towards the waveguide surface as the external voltage decreases. In the transmission measurements, the high numerical aperture fibre always collects the entire output mode independently of its vertical position owing to the electro-optical effect.

\section{Conclusion}

A novel and highly performing PC light modulator fabricated on lithium niobate is reported. We use the light propagation inside the PC combined to a non-linear substrate material to achieve an enhancement of the electro-optical effect. It consists of a square lattice of air holes in LN milled by FIB on an APE lithium niobate waveguide surrounded by two parallel electrodes.

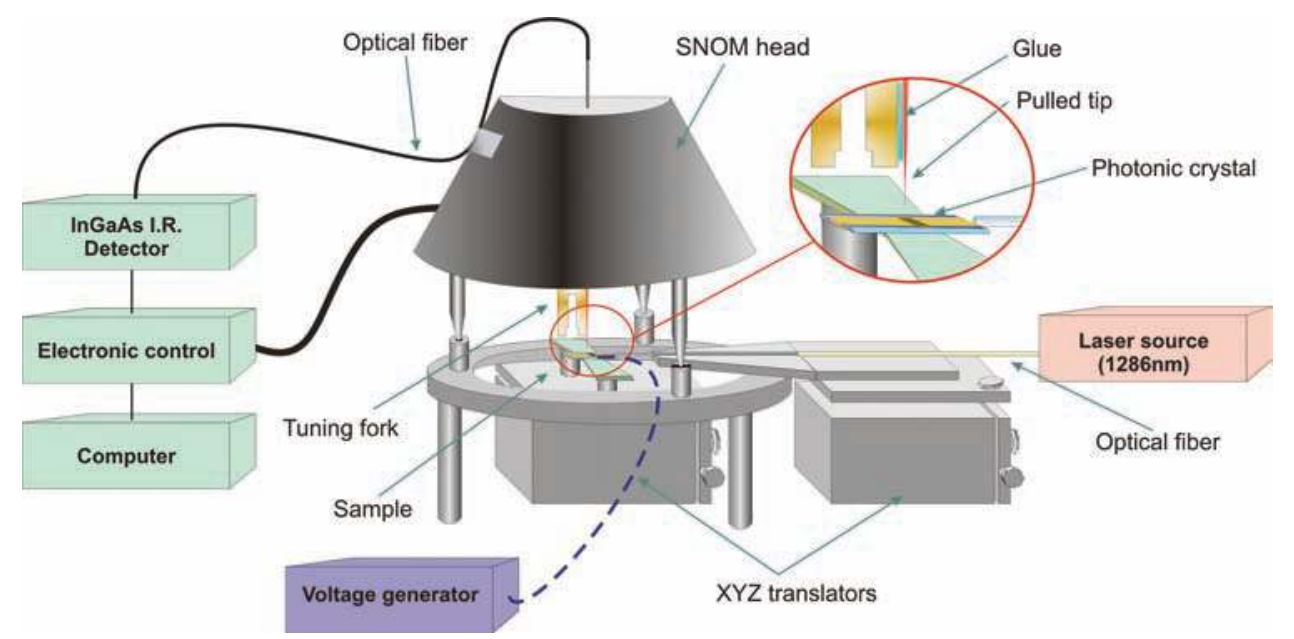

Fig. 4. 3D representation of the scanning near-field optical set-up. 

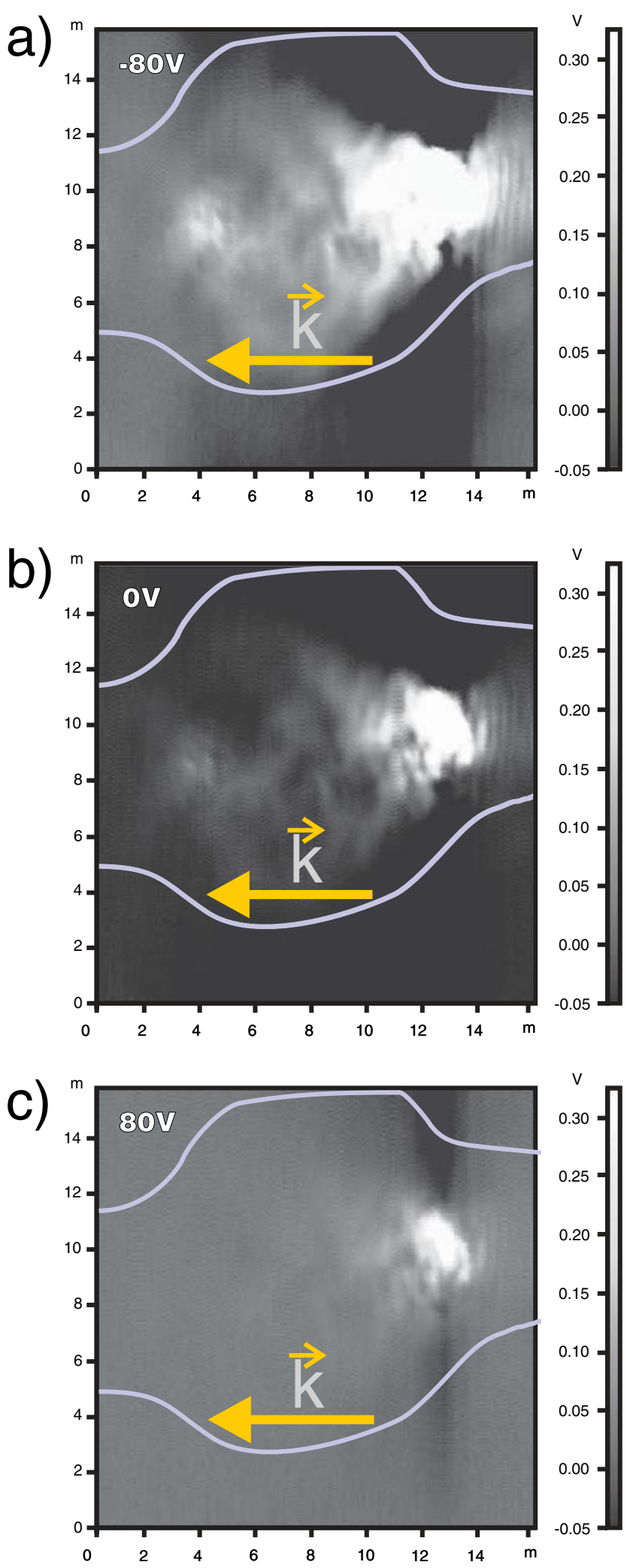

Fig. 5. SNOM images of the photonic crystal at three different voltages: (a) $-80 \mathrm{~V}$, (b) $0 \mathrm{~V}$ and (c) $80 \mathrm{~V}$. The blue line represents the contour of the photonic crystal and of the waveguide.
Transmission measurements show tunability 300 times bigger than for a classical device. Finite difference time domain simulations have allowed us to calculate the local field factor and subsequently the effective refractive index difference showing an excellent agreement between theory and experiment. The measured enhanced electro-optical effect has been confirmed by optical near-field images performed with an SNOM in collection mode at $1286 \mathrm{~nm}$ and three external excitation voltages $-80,0$ and $80 \mathrm{~V}$.

Work is in progress to fabricate a high-speed modulator of the same characteristics.

\section{Acknowledgements}

This work has been supported by the Action Concertée Incitative 'Nanosciences' COBIAN, No. NR 137. The authors thank the Technological Central MIMENTO at FEMTO-ST for technical support.

\section{References}

Bernal, M.-P., Courjal, N., Amet, J., Roussey, M. \& Hou, C.H. (2006) Lithium niobate photonic crystal waveguides: far field and near field characterisation. Opt. Commun. 265, 180-186.

Delaye, P., Astic, M., Frey, R. \& Roosen, G. (2005) Transfer-matrix modelling of four-wave mixing at the band edge of a one-dimensional photonic crystal. J. Opt. Soc. Am. B 22, 2492-2504.

Flück, E., Hammer, M., Otter, A.M., Korterik, J.P., Kuipers, L. \& van Hulst, N.F. (2003) Amplitude and phase evolution of optical fields inside periodic photonic structures. J. Llightwave Technol. 21, 1384-1393.

Gérard, D., Berguiga, L., de Fornel, F., et al. (2002) Near-field probing of active photonic-crystal structures. Opt. Lett. 27, 173-175.

Gersen, H., Karle, T.J., Engelen, R.J.P., et al. (2005) Real-space observation of ultraslow light in photonic crystal waveguides. Phys. Rev. Lett. 94, 073903.

Koenderink, A.F., Wüest, R., Buchler, B.C., et al. (2005) Near-field optics and control of photonic crystals. Photon. Nanostruct. Fundam. Appl. 3, 63-74.

Lacour, F., Courjal, N., Bernal, M.-P., Sabac, A., Bainier, C. \& Spajer, M. (2005) Nanostructuring lithium niobate substrates by focused ion beam milling. Opt. Mater. 27, 1421-1427.

Notomi, J.M., Yamada, K., Shinya, A., Takahasi, J., Takahasi, C. \& Yokohama, I. (2001) Extremely large group-velocity dispersion of line-defect waveguides in photonic crystal slabs. Phys. Rev. Lett. 87, 253902.

Razzar, L., Träger, D., Astic, M., Delaye, P., Frey, R. \& Roosen, G. (2005) Kerr and four-wave mixing spectroscopy at the band edge of one-dimensional photonic crystals. Appl. Phys. Lett. 86, 231106.

Rigneault, H., Lourtioz, J.-M., Delalande, C. \& Levenson, A. (2005) La nanophotonique. Hermes Science, Lavoisier.

Roussey, M., Bernal, M.-P., Courjal, N. \& Baida, F.I. (2005) Experimental and theoretical characterization of a lithium niobate photonic crystal. Appl. Phys. Lett. 87, 241101.

Schneider, G.J. \& Watson, G.H. (2003) Nonlinear optical spectroscopy in one-dimensional photonic crystals. Appl. Phys. Lett. 83, 5350-5352. 
Soljaci, M. \& Joannopoulos, J.D. (2004) Enhancement of nonlinear effects using photonic crystals. Nat. Mater. 3, 211-219.

Soljaci, M., Johnson, S.G., Fan, S., Ibanescu, M., Ippen, E. \& Joannopoulos, J.D. (2002) Photonic-crystal slow enhancement of nonlinear phase sensitivity. J. Opt. Soc. Am. B 19, 2052-2059.

Vlasov, Y.A., O'Boyle, M., Hamann, H.F. \& McNab, S.J. (2005) Active control of slow light on a chip with photonic crystal waveguides. Nature 438, 65-69. 